\title{
Rapid diagnosis of glucocorticoid suppressible hyperaldosteronism in infants and adolescents
}

\author{
A Jamieson, G C Inglis, M Campbell, R Fraser, J M C Connell
}

\begin{abstract}
Glucocorticoid suppressible hyperaldosteronism (GSH) is an uncommon form of dominantly inherited hypertension. Presentation with hypertension and complications such as stroke in early life are well recognised. The use of a simple genetic test carried out on blood or placenta facilitates the detection of infants and children with GSH before the development of hypertension, allowing prompt treatment of hypertension if it occurs, and an opportunity to study the effects of growth and environmental influences on the progression of the condition. (Arch Dis Child 1994; 71: 40-43)
\end{abstract}

Hypertension in childhood is uncommon and secondary causes such as underlying diseases of the kidney, coarctation of the aorta, or adrenal enzyme deficiency must be excluded. Glucocorticoid suppressible hyperaldosteronism (GSH) is such a secondary disorder which can cause severe hypertension in young children. Presentation of GSH with acute renal failure ${ }^{1}$ or as a cerebrovascular accident in adolescents or young adults has been described. In many cases the disorder is not diagnosed until adult life when hypertension may be severe and associated with end organ damage such as left ventricular hypertrophy. The disorder is characterised by an autosomal dominant form of inheritance and features of hyperaldosteronism - that is, increased plasma aldosterone concentrations, suppressed plasma renin concentration, often a low plasma potassium concentration, and hypertension. No adrenal masses are found in GSH and administration of exogenous glucocorticoids, usually dexamethasone $2 \mathrm{mg} /$ day for two to four weeks results in a total reversal of these abnormalities. ${ }^{2}$ In addition, a number of physiological and biochemical features peculiar to GSH have been described. These include loss of the normal aldosterone response to angiotensin II infusion, excretion of large amounts of the unusual 18-hydroxylated steroids 18-hydroxycortisol and 18-oxocortisol in urine, and an increase in plasma aldosterone, 18-hydroxycortisol, and 18-oxocortisol levels in response to corticotrophin (ACTH) infusion, all of which are reversed by long term treatment with glucocorticoids by mouth. ${ }^{2}$

The molecular pathology underlying GSH has been shown to be the presence of a chimaeric 11 $\beta$-hydroxylase/aldosterone
Department of Medicine and Therapeutics, Western Infirmary, Glasgow G11 6NT A Jamieson

MRC Blood Pressure Unit G C Inglis M Campbell R Fraser J M C Connell

Correspondence to: Dr Jamieson.

Accepted 6 April 1994
Crossing over at meiosis between the 11ß-hydroxylase and aldosterone synthase genes

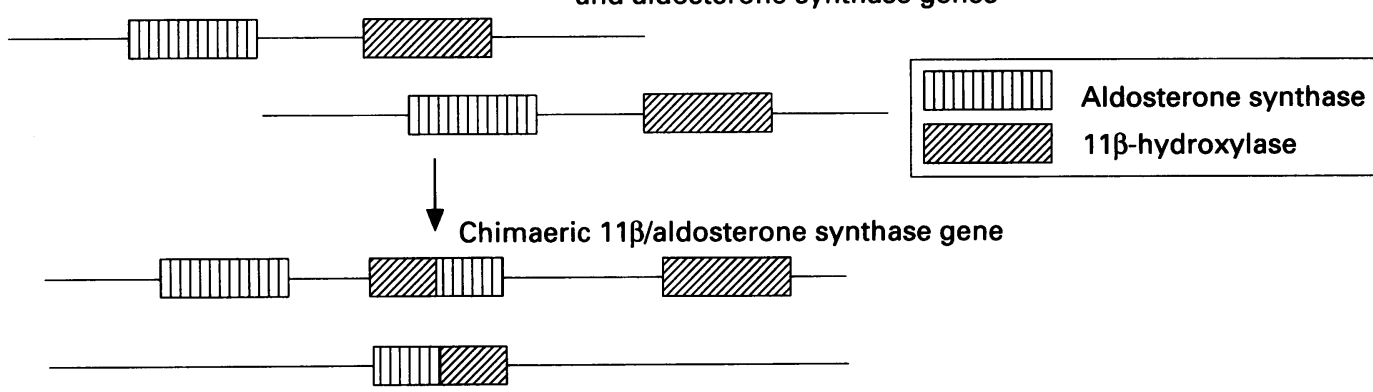

Unequal crossing over at meiosis results in a chimaeric gene with a 5 ' ACTH responsive regulatory sequence encoding an enzyme with aldosterone synthase activity and another gene with $11 \beta$-hydroxylase activity whose expression is regulated by angiotensin II. The activity of this other gene is unknown as yet

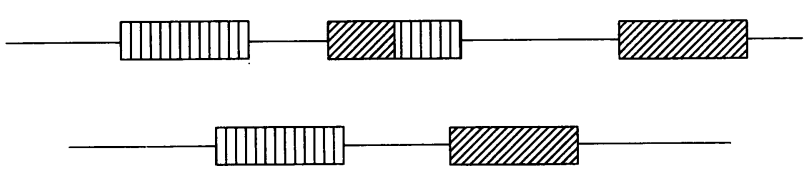

Genotype of an affected individual.

The chimaeric $11 \beta /$ aldosterone synthase gene is transmitted to affected individuals who become heterozygous for the chimaeric gene

Figure 1 Schematic representation of the formation of a chimaeric 11 $\beta$-hydroxylase/aldosterone synthase gene. Affected subjects thus have five genes contributing to $11 \beta$-hydroxylase or aldosterone synthase activity, four 'normal' genes, and an extra chimaeric gene. 

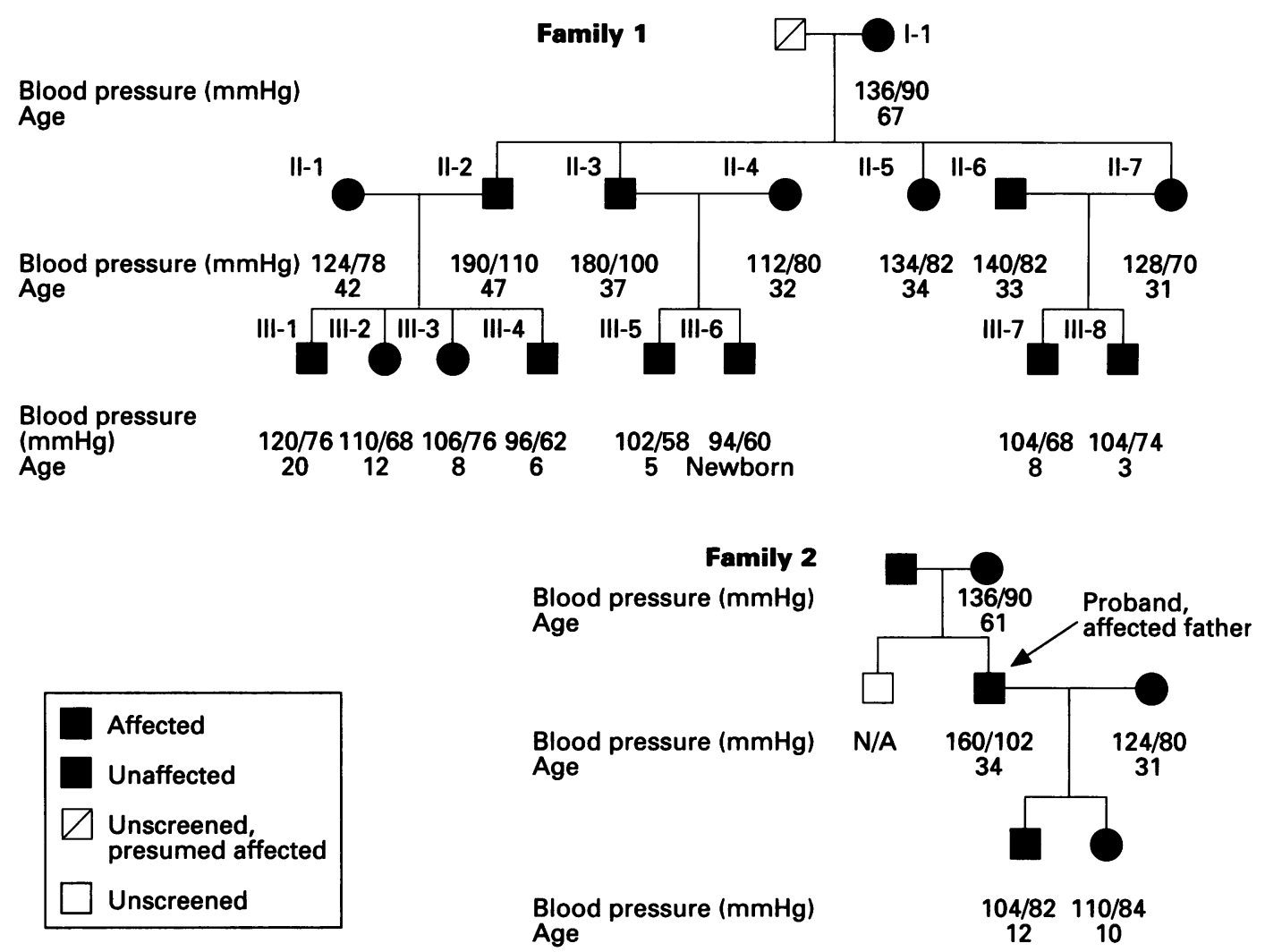

Figure 2 Family trees of the two families studied. Blood pressure measured at rest while receiving no treatment. Family 1: each generation is marked as either I (grandparent), II (parent), or III (child). Subject III-6 is the infant whose placental DNA was studied. Family 2: proband father is identified by an arrow. Other members of the family are described in fig 3.

synthase gene in affected subjects. ${ }^{3}$ Such a gene combines most of the coding sequence of the gene for aldosterone synthase (CYP11B2) with the $5^{\prime}$ end of the gene for $11 \beta$-hydroxylase (CYP11B1) containing the promoter sequence of the gene for 11ß-hydroxylase (fig 1). The promoter region of the $11 \beta$-hydroxylase gene contains an ACTH responsive element which positively regulates the expression of this gene in the adrenal gland and the chimaeric gene encodes an enzyme capable of aldosterone synthesis in the zonae glomerulosa and fasciculata, but whose expression is regulated by ACTH - that is, aldosterone synthesis is regulated by ACTH and suppressed by factors lowering plasma ACTH concentrations such as glucocorticoids. The presence of such a chimaeric gene explains all the biochemical and clinical features of GSH and has been found in all patients with GSH examined so far. ${ }^{45}$ The chimaeric gene results in a restriction fragment length polymorphism (RFLP) when genomic DNA from affected subjects is digested with the restriction enzymes BamHI or EcoRI and probed with a CYP11B1 specific probe. In normal subjects genomic DNA digested with the enzyme BamHI results in the presence of two bands on the resultant autoradiograph, one for 11ß-hydroxylase and one for aldosterone synthase. In subjects with GSH a third band due to the chimaeric gene is present. The presence of this RFLP has been shown to be $100 \%$ specific for the diagnosis of $\mathrm{GSH}^{5}$ and as reliable and more convenient than the measurement of steroids in plasma or urine. ${ }^{6}$
We describe the use of RFLP patterns in the rapid diagnosis of GSH in the perinatal period and in childhood in the children of subjects with GSH and discuss the implications of the early diagnosis of this disorder.

\section{Methods}

The father of the children in family 1 (II-3) described in the following was the subject of a previous study and his features and those of his siblings have been described elsewhere ${ }^{7}$; the children of family 1 (III-1 to 8) have not been formally studied until now. The proband of family 2 was diagnosed as having GSH on the basis of blood pressure increased above two standard deviations of the normal value for age and sex, hyperaldosteronism with suppressed plasma renin concentration, and reversal of all three features after treatment with dexamethasone. The features of this family have not been reported previously.

Peripheral blood was obtained from all available members of family 1 and family 2 . Placental tissue from the younger son in family 1 (III-6) was obtained at birth and rapidly frozen in liquid nitrogen and stored at $-70^{\circ} \mathrm{C}$. DNA was extracted from blood and placental tissue using a standard method and stored in TRIS-EDTA buffer at $4^{\circ} \mathrm{C} .{ }^{4}$ Ten micrograms of DNA was digested using high concentration BamHI $(50 \mathrm{U} / \mu \mathrm{l})$ (Gibco BRL) overnight at $37^{\circ} \mathrm{C}$ and then subjected to electrophoresis in a $0.8 \%$ agarose gel. DNA was blotted on to a nylon membrane (Hybond $\mathrm{N}$, Amersham) using a modified version of the Southern 

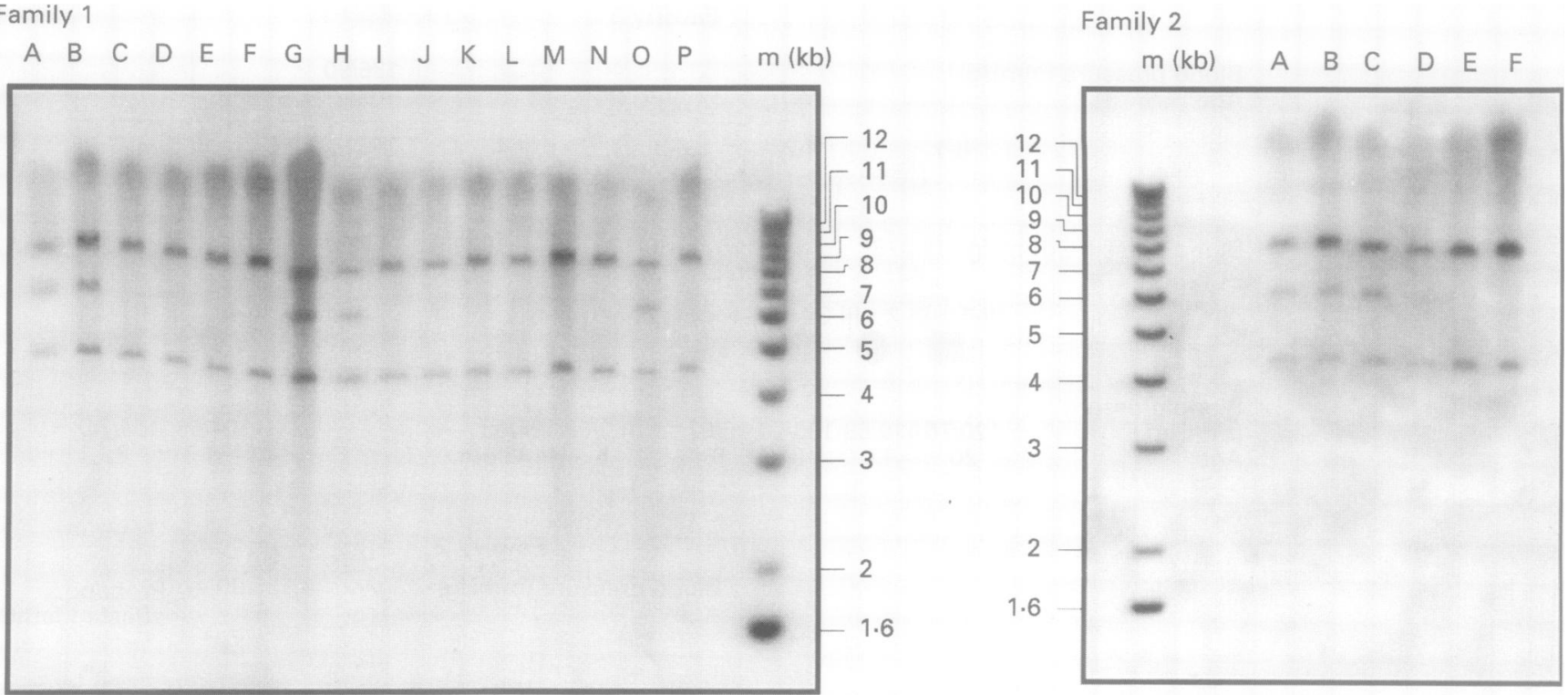

Figure 3 Results of probing BamHI digested DNA with a CYP11B1 exon 3-5 probe. Affected subjects have three hybridising species: an 8.5 kb species corresponding to CYP11B1, a $4.5 \mathrm{~kb}$ species corresponding to CYP11B2, and a third $6.3 \mathrm{~kb}$ band corresponding to the chimaeric $11 \beta$ aldosterone synthase gene. $m=$ Size marker. Family 1: Five affected subjects in two generations, suggesting the deceased grandparent was the carrier of the mutation. $A=I I-2 ; B=I I-3 ; G=I I I-1 ; H=I I I-2 ;$ and $O=I I I-6$, affected carriers of $G S H$. All others are unaffected. Family $2: A=$ affected proband; $B=$ daughter of proband; $C=$ mother of proband; $D=$ unaffected father of proband; $E=$ unaffected wife of proband; and $F=u n a f f e c t e d$ son of proband.

blotting technique. ${ }^{4}$ Membranes were then baked at $65^{\circ} \mathrm{C}$ for five hours. A probe was prepared from a 500 base pair fragment encompassing exons 3-5 of CYP11B1 derived from the polymerase chain reaction (PCR) amplification of CYP11B1 (primer 1-5', AGAAAATCCCTCCCCCCTA; primer 2-5', GACACGTGGGCGCCGTGTGA), labelled with phosphorus-32 using the random primer method, and hybridised overnight at $65^{\circ} \mathrm{C} .^{3}$ After washing in $1 \mathrm{XSSC} / 0.5 \%$ sodium dodecylsulphate, autoradiographs were prepared by placing the membranes in contact with Kodak Hyperfilm for five days at $-70^{\circ} \mathrm{C}$ and developing in a Kodak X-Omat.

\section{Results}

Figure 2 shows the family tree with blood pressure data for the two families. Figure 3 shows the results of probing DNA digested with BamHI from the family members.

In family 1, RFLP analysis shows that both II-2 and II-3 are carriers of the chimaeric GSH gene. With reference to the family of the neonate, the mother (II-4) is unaffected, the older son (III-5) is unaffected, and the newborn son (III-6) is affected. Analysis of the other members of the kindred reveal two other affected subjects, III-1 and III-2, both of whom have normal blood pressure. Subsequent biochemical data (not reported) show increased plasma aldosterone concentrations with suppressed plasma renin.

In family 2, RFLP analysis allows the identification of a young child with GSH and normal blood pressure and the autosomal dominant pattern of inheritance in this family.

\section{Discussion}

Making a diagnosis of GSH in an individual subject allows appropriate treatment to be instituted in affected subjects and also signals the need to screen other family members for the presence of GSH. In adults, this was previously based on the results of 24 hour aldosterone profiles, aldosterone response to ACTH and angiotensin II infusions, and 18-hydroxycortisol excretion before and after dexamethasone treatment. ${ }^{6}$ These methods are limited, however, by the time intensive investigations and the limited availability of many of the assays; they can now be supplanted in affected subjects by the use of a simple genetic test on a small blood or tissue sample.

Clearly the question of when to start treatment is difficult and at present we measure blood pressure biannually in all affected subjects to allow early detection of an increase in blood pressure above that expected for their age and sex. In adults the commonly used treatments are dexamethasone, amiloride, and spironolactone. Dexamethasone has excellent effects on the biochemical and clinical features of GSH but in theory could retard growth and bone development; we have therefore avoided its use in children. Similarly, spironolactone, an aldosterone antagonist, can lower blood pressure and reverse hypokalaemia in GSH but its antiandrogenic properties preclude its use in prepubertal children. In those children who develop hypertension as a feature of GSH we recommend the use of amiloride in a dose titrated to achieve optimum blood pressure control - that is, 5-10 mg daily. Once a subject is identified as having GSH then screening of relatives is a simple matter, requiring only a single blood sample and a measurement of blood pressure.

Our study shows that a perinatal diagnosis of GSH can be confirmed or excluded by the use of placental tissue for DNA extraction. Follow up and treatment of subjects with GSH at an appropriate time with the hope of 
preventing the potential childhood morbidity and mortality of the disorder is thus made possible. In addition, it is obvious that there are marked phenotypic differences in the same kindred and early diagnosis of GSH allows the prospective study of affected subjects to determine the effects of environment, growth, and sexual maturation on the phenotypic expression of the disease. Studies of this nature are underway, as are the characterisation of a further four kindreds with GSH to determine the role of dietary and other genetic factors on the evolution of the disorder.

A Jamieson is a Medical Research Council Training Fellow.

1 Lee SM, Lightner E, Witte M, Oberfield S, Levine L, New MI. Dexamethasone suppressible hyperaldosteronism in a child with nephrosclerosis. Acta Endocrinol (Copenh) 1982; 99: 251-5.

2 Jamieson A, Connell J, Fraser R. Glucocorticoid-suppressible hyperaldosteronism: from confusion to conclusion? f Mol Endocrinol 1993; 10: 3-5.

3 Lifton R, Dluhy R, Powers M, et al. A chimaeric 11 betahydroxylase/aldosterone synthase gene causes glucocorticoid-remediable aldosteronism and human hypertension. Nature 1992; 335: 262-5.

Nature 1992; 335: 262-5.
4 Pascoe L, Curnow K, Slutsker L, et al. Glucocorticoid suppressible hyperaldosteronism results from hybrid genes
super created by unequal crossovers between CYP11B1 and CYP11B2. Proc Natl Acad Sci USA 1992; 89: 8327-31.

5 Lifton RP, Dluhy RG, Powers $M$, et al. Hereditary hypertension caused by chimaeric gene duplications and ectopic expression of aldosterone synthase. Nature Genetics 1992; 2: 66-74.

6 Rich GM, Ulick S, Cook S, Wang JZ, Lifton RP, Dluhy RG. Glucocorticoid-remediable aldosteronism in a large kindred: clinical spectrum and diagnosis using a characteristic biochemical phenotype. Ann Intern Med 1992; 116: 813-20.

7 Connell J, Kenyon C, Corrie J, Fraser R, Watt R, Lever A Dexamethasone-suppressible hyperaldosteronism. Adrenal transition cell hyperplasia? Hypertension 1986; 8: 669-76. 